\title{
Optical Transmission Schemes for GEO Feeder Links
}

\author{
William Cowley \\ University of South Australia \\ bill.cowley@unisa.edu.au
}

\author{
Dirk Giggenbach \\ German Aerospace Center \\ dirk.giggenbach@dlr.de
}

\author{
Ramon Mata Calvo \\ German Aerospace Center \\ ramon.matacalvo@dlr.de
}

\begin{abstract}
A novel transmission scheme for the forward link of a broadband satellite system that aims to avoid the need for feeder-link RF spectrum is described ${ }^{1}$. The hybrid scheme uses one or more optical uplinks from a hub station to geostationary satellite, plus one or more Ka band downlinks. The approach aims to retain the advantages of strong channel coding with high spectral efficiency, as used in recent high-speed digital video broadcast satellites, yet minimise on-board processing requirements. Promising simulation results are presented for a range of channel models, including channel measurements made with ESA's Artemis satellite.
\end{abstract}

\section{INTRODUCTION}

High bandwidth satellite links using DVB-like transmission schemes are now widely used for a range of communication services. However geostationary satellite systems providing broadcast services and broadband internet to remote areas find increasing difficulty in obtaining scarce radio frequency spectrum, both for feeder links between hub stations and GEO satellites and also links between satellites and small terminals.

Free space optical transmission can provide Gbit data rates to geostationary orbit and avoid the use of any RF spectrum but may suffer in turn from attenuation due to clouds and scintillation fading. Hybrid schemes using both optical and RF transmission have good potential, provided low-complexity transponders can be devised. In this paper, one or

\footnotetext{
${ }^{1}$ This study has been supported by the German Academic Exchange Service.
}

more optical uplinks from hub earth station to a GEO satellite are considered, with an RF downlink to a ground terminal. We aim to avoid the use of RF spectrum in the feeder uplink and retain the coding and spectral efficiency benefits (e.g. [1]) of a DVB-S2 type of transmission scheme as far as possible. Our approach is designed to avoid the high complexity of a fully regenerative payload which will not be feasible, especially at the data rates envisaged in these applications.

The paper is organised as follows. Propagation characteristics of both the optical uplink and the downlink channel models are crucial in the design of efficient hybrid transmission schemes. These models are consider in Section II. In Section III we describe the hybrid transmission system, including various coding and interleaving options. We present results in Section IV then finish with conclusions.

\section{Channel Models}

\section{A. Uplink}

Free space optical (FSO) links between ground and space can use either coherent or non-coherent approaches[4][5][6]. While the former are gaining in popularity, links using intensity modulation and "direct detection" are still much more common. This paper assumes the latter scenario, generally using on-off keyed (OOK) modulation, although the noise model could be readily adapted to cater for coherent FSO approaches.

FSO links suffer channel effects in two broad areas. First, in addition to free space loss the signal can be attenuated by fog, clouds, dust etc. In the GEO feeder link scenario this is a significant issue 


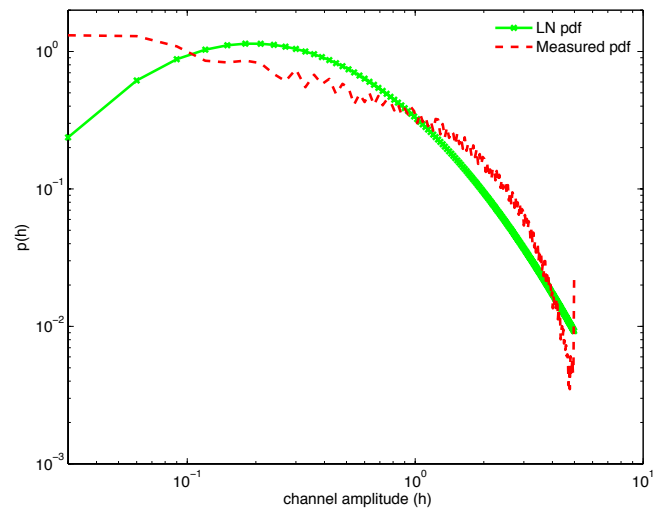

Fig. 1. Log-normal and measured fading in FSO GEO Uplink.

but can be addressed by the use of multiple optical ground stations (OGS) in geographically separated regions [7]. Secondly FSO links are impaired from fading caused by small index-of-refraction fluctuations in the atmosphere. This scintillation fading is typically modelled by log-normal (LN) or gammagamma fading distributions of the FSO channel gain $h$. The normalised irradiance variance, usually denoted by $\sigma_{I}^{2}$ and called Scintillation Index (SI), provides a simple measure of fading severity.

$$
\sigma_{I}^{2}=\frac{E\left\{h^{2}\right\}}{E\{h\}^{2}}-1
$$

In a GEO link, the atmosphere is located very close to the OGS. In the uplink, the scintillation, produced by the atmospheric turbulence, generates an intensity speckle pattern in far-field, with bright and dark flecks. The speckle size is bigger than the satellite telescope and therefore the received power by the photo-detector drops dramatically when a dark area of the received beam illuminates the telescope. Moreover, pointing errors due to the remaining beam wander lead to additional deep fades. On the contrary, in downlink, the speckle size of the received beam is generally smaller than the receiver aperture and furthermore bigger telescopes are used. When the telescope size is larger than the scintillation pattern, the power fluctuations decrease on the detector due to the so-called aperture averaging effect. Therefore, uplink fading is significantly worse in such scenario than downlink fading [3].

Artemis, a GEO satellite developed and operated by ESA, demonstrated in 2001 the feasibility and performance of free-space optical inter-satellite links from a LEO satellite to a geostationary relay, and bidirectional links between a GEO and ESA's optical ground station located on Tenerife (Spain). In 2012 and 2013, DLR together with Cassidian Optronics under ESA Contract (ESA contract 4000104838/11NL/US) performed two measurement campaigns with the Artemis bidirectional GEO-GND link system. The scope was to characterise the uplink fading channel and the effectiveness of transmitter diversity for fading mitigation.

This paper has used a small number of Artemis uplink channel measurements [2] to help model the uplink channel. Figure 1 illustrates a highscintillation fading distribution from Artemis, with SI close to unity. An LN distribution with $\mathrm{SI}=2$ is shown for comparison ${ }^{2}$. The figure shows that $\mathrm{LN}$ distributions do not always provide a good model for Artemis measurements. On the other hand, lowSI Artemis measurements are a better match to LN. In Section IV both Artemis measurements and LN distributions have been used to simulate the transmission schemes outlined below.

In the following section we assume the optical irradiance is modulated, either digitally using onoff keying or via analog approaches. The resulting electrical signal after the photodetector in the OOK case is given by

$$
y_{k}=h b_{k}+\nu_{k}
$$

where $b_{k}$ is the $k$-th OOK bit and the noise term is assumed Normally distributed, $\nu \sim N\left(0, \sigma_{o}^{2}\right)$. In this normalised model, the OOK sample $y_{k}$, after DC removal, can be assumed to be \pm 1 . Using $E\{h\}=1$, the average uplink electrical SNR is:

$$
\gamma_{u}=E\left\{h^{2}\right\} / \sigma_{o}^{2}=\left(1+\sigma_{I}^{2}\right) / \sigma_{o}^{2}
$$

Fades typically last for milliseconds, which is long compared to DVB codewords lengths. Mitiga-

\footnotetext{
${ }^{2}$ It can be readily shown that if $E\{h\}=1$, the mean and variance parameters of the underlying $\mathrm{LN}$ distribution are given by $\sigma_{L N}^{2}=\log \left(1+\sigma_{I}^{2}\right)$ and $\mu_{L N}=-\sigma_{L N}^{2} / 2$.
} 
tion strategies to deal with the relatively long fade duration are considered below.

\section{B. Downlink}

Short-term RF downlink fading is typically much smaller than the uplink optical fading described above. Attenuation due to rain is an issue in $\mathrm{Ka}$ Band applications but has not been addressed in this paper. In addition, for simplicity, the transponder $\mathrm{RF}$ PA is assumed to be linear. The $\mathrm{Ka}$ Band downlink is therefore modelled as AWGN with DVB-S2 LDPC channel coding.

Assuming an overall downlink channel gain factor of $g$, discussed below, the $j$-th received symbol sample, after matched filtering will be

$$
r_{j}=g a_{j}+n_{j}
$$

where $a_{j}$ is the $j-$ th RF symbol. The RF downlink rate is likely to be at least $100 \mathrm{Mbit} / \mathrm{s}$. However the optical uplink could be much faster, so for digital schemes, one uplink might carry multiplexed downlinks.

\section{HYBRID TRANSMISSION OPTIONS}

\section{A. Uplink Options}

In a simple "radio over optical" scenario the modulated DVB signal (say at a suitable IF) is used to amplitude modulate an optical source. We neglect linearity issues or efficiency losses. Irradiance variations due to fading are imposed on the downlink RF signal (unless AGC is used). Alternatively the uplink could carry digital information such as encoded bits from the DVB-S2 channel encoder, modulated as OOK pulses. These schemes could be used with or without interleaving. If there is no interleaving, due to the high data rates, each codeword is affected by one value of channel amplitude.

As mentioned above, multiple uplinks could be available to mitigate the effect of clouds. With careful bit-level synchronisation these could also provide diversity gain. Alternatively one cloudless OGS could achieve diversity by duplication of optical sources separated by a small distance, or by repeating the modulated uplink signal through the same optics but at a different wavelength, after say 20 msecs, assuming appropriate on-board storage.

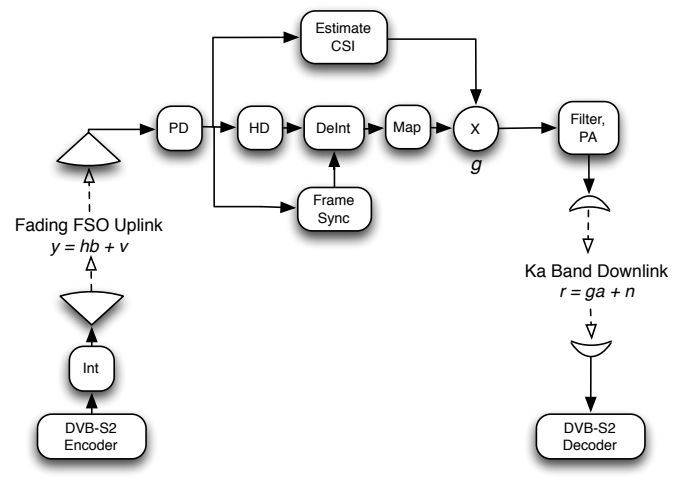

Fig. 2. System Model for FSO GEO Feeder Simulations, assuming OOK Uplink with Interleaving, plus RF downlink.

In these cases various forms of diversity combining are possible and provide useful improvements for uncorrelated uplink fading. In the next section we compare the single-uplink case to dual-uplinks, with both selection diversity and equal gain combining.

\section{B. Interleaving}

Without interleaving, codewords will be lost during optical fades. This implies that a high uplink SNR and/or low level of fading will be required for satisfactory performance. Interleaving over tens of milliseconds in the optical uplink allows each codeword to experience the full range of channel fading values. With 100Mbit/s and 10k codewords, a 2D interleaver might require (say) 200 codewords (2 Mbit) for an interleave depth of 20 msecs. Alternatively, the optical stream might contain many DVB streams and so require a larger interleaver.

Figure 2 shows a model of the uplink, with interleaving. In the satellite, after the photo-detector the symbol samples are converted to "hard-decisions", then de-interleaved. The resulting bits are mapped to QAM or APSK symbols. Before conventional pulse shaping the symbol amplitude is adjusted, as described below.

Interleaving could be carried out on individual bits, or groups of bits. Since the impact of optical fades should be limited to the least number of RF symbols, we have assumed that groups of $M$ bits, all belonging to one symbol, are interleaved. Thus 
for 16QAM, each 4 bits mapped to one symbol will all experience the same value of $h$. In practice the deinterleaver might represent the most demanding aspect of on-board processing. Robust frame synchronisation could be achieved by the insertion of regular unique word bit patterns, with a UW correlator that "freewheels" through channel fades. We believe this processing would still be significantly simpler than a fully regenerative payload.

\section{Uplink Errors}

In digital schemes with interleaving, some symbols from the on-board mapper are corrupted by uplink bit errors. This fraction depends on the optical channel scintillation and equivalent uplink noise level. Even a small fraction of incorrect symbols must be handled in some manner or they cause the LDPC decoder to fail.

If these corrupt symbols can be identified, the impact of uplink errors can be greatly reduced. A simple approach is to set their amplitude to zero by controlling the gain factor $g$ on a symbol by symbol basis. Tests confirm that a small fraction of "erasures" (e.g. up to 20\%) has a relatively modest effect on a powerful rate-1/2 channel code. In practice it may not be trivial to identify the RF symbols that have been affected by uplink bit errors. In the following the optical signal level is compared to an erasure threshold and corresponding RF symbols erased if $h$ is less than this threshold. An obvious extension is to use a simple code on the uplink bits to help detect errors. For example a single parity check code could be used, either for the bits in one symbol, or in two dimensions for a small number of symbols. A 1D SPC scheme has been simulated in the next section.

\section{Channel State Information and Demapping}

Ideally the DVB decoder in the ground terminal should know which symbols have been deleted. Use of a standard demapper that assumes all RF symbols are of equal quality gives poor results.

One option is to multiplex CSI (channel state information) into the RF downlink. Another option is to modify the demapper in the downlink receiver so that it knows some RF symbols may be erased, but not send downlink specific information regarding symbol erasures. For example, using a MAP approach suppose we wish to maximise the probability of symbol $a$ given sample value $r$, i.e. $P(a \mid r)$. Treating the downlink channel amplitude as a discrete random variable with values $g_{i}, i=0$ or 1 , we select $a$ to maximise

$$
\sum_{i} P\left(a, g_{i} \mid r\right) \propto \sum_{i} P\left(r \mid a, g_{i}\right) P(a) P\left(g_{i}\right)
$$

With just two values of $g$, equal to 0 or 1 , and equiprobable symbols, this gives

$$
\text { maximise } P\left(r \mid a, g_{0}\right) P\left(g_{0}\right)+P\left(r \mid a, g_{1}\right) P\left(g_{1}\right)
$$

Letting the erasure probability $P_{e}=P\left(g_{0}\right)$ gives a modified likelihood metric of

$\Lambda(a)=\frac{P_{e}}{\sqrt{2 \pi \sigma_{n}^{2}}} e^{-\|r\|^{2} / 2 \sigma_{n}^{2}}+\frac{1-P_{e}}{\sqrt{2 \pi \sigma_{n}^{2}}} e^{-\|r-a\|^{2} / 2 \sigma_{n}^{2}}$

In addition to this metric a sub-optimum version has been tried, that instead of using CSI in the decoder just selects the maximum of the two terms in (5). Even this simple method yields significant improvements in the DVB-S2 decoder performance compared to assuming that the channel is always unity.

\section{RESUlTS}

This section provides initial results of Monte Carlo simulations of the hybrid transmission schemes described above, including interleaving, symbol erasure based on fade level and possibly per-symbol SPC, plus the blind decoding metric outlined in (5). LDPC routines from [8] been used, plus custom code. Most simulations used 1/2 rate, 16k codewords. Each Monte Carlo simulation used at least 500 codewords for each value of downlink SNR. Simulations with interleaving have used IID uplink channel samples (i.e. assuming a large interleaver).

Figure 3 shows that without interleaving the performance is poor. In this figure the uplink SNR, $\gamma_{u}$, is $16 \mathrm{~dB}$, with $\mathrm{LN}$ fading of $\sigma_{I}^{2}=0.5$ and 16QAM used on the downlink. Analog and digital (i.e. OOK) schemes, with and without diversity, but no interleaving, are compared. The analog schemes 


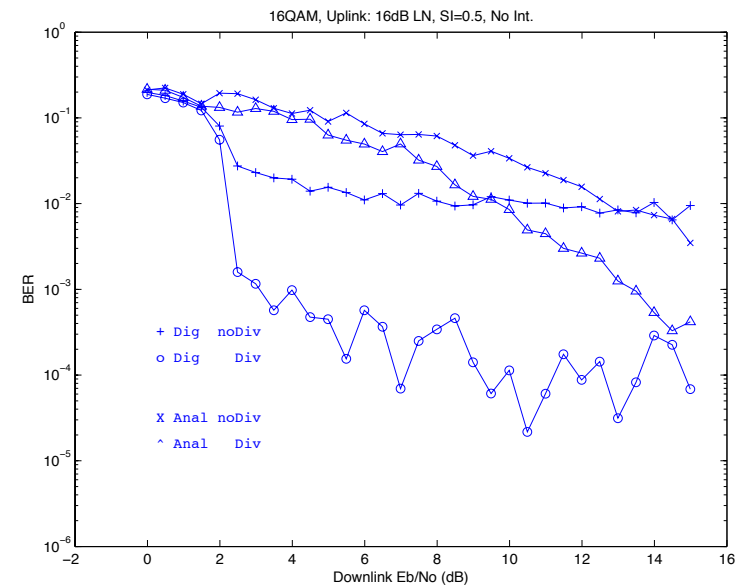

Fig. 3. BER after LDPC decoding, for analog and digital uplinks without interleaving.

perform worse than the digital approach. Ideal gain control is used in the analog methods so that when the optical signal fades the downlink RF will be constant average amplitude but dominated by the effect of uplink noise. (Simulations without AGC gave fairly similar results.) The plots with diversity give some improvement but not enough for reasonable performance.

Figure 4 shows much better 16QAM performance using digital schemes with interleaving, under $\sigma_{I}^{2}=$ $0.5 \mathrm{LN}$ fading conditions, with and without diversity in the uplink. The processing corresponds to Figure 2 and no SPC check was used in this case. The right-hand plots show that as the uplink SNR is increased from 8 to $12 \mathrm{~dB}$ the singleuplink performance improves dramatically. The lefthand (solid) plots illustrate a significant gain from diversity using dual uplink channels. Equal gain combining was used in these results and generally seems to offer modest gains over selection diversity.

Figure 5 shows sample simulation results for 32APSK at a fairly low uplink SNR but with low levels of SI fading. The figure compares the decoder with CSI to the "blind CSI" metric of (5). Some loss in performance is evident with the blind metric. The figure also illustrates the benefit of using uplink diversity compared to a single uplink.

An error floor is visible in some of these plots.

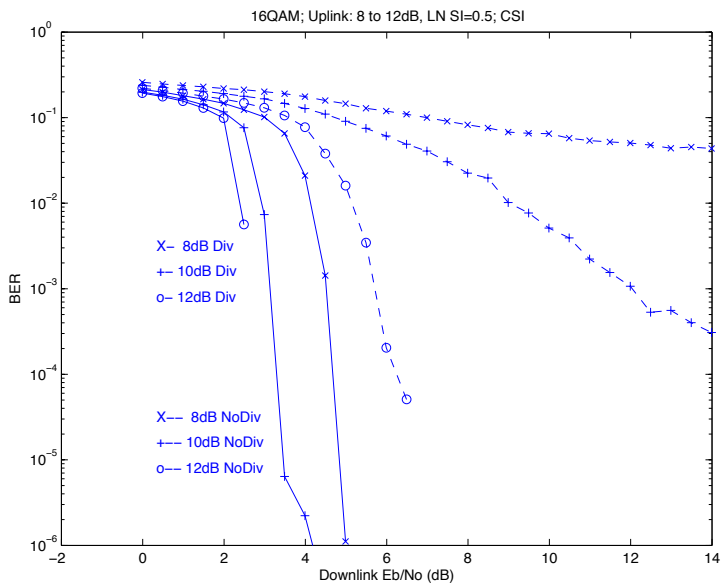

Fig. 4. Performance with interleaving and CSI, over a range of uplink SNRs.

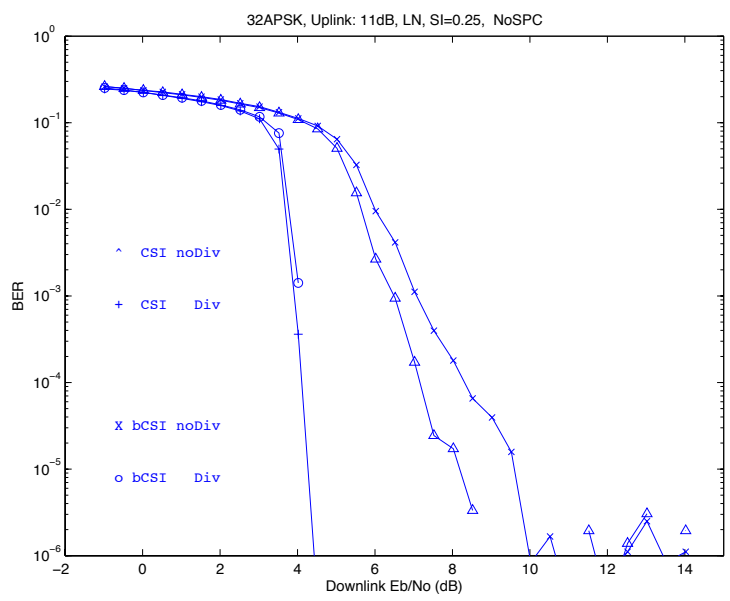

Fig. 5. 32APSK Simulations at $11 \mathrm{~dB}$ uplink SNR, with different decoder metrics.

DVB-S2 usually uses LDPC concatenated with a BCH code to "mop up" errors remaining from the LDPC. Note that the BCH code has not been used in the present simulations. The error floor observed in these plots is worse than the usual performance of this code in an AWGN channel, but could still be repaired with a high-rate outer code, or by custom design of the LDPC code. In any case, careful adjustment of the erasure threshold was needed to achieve the current results. In general it was found 


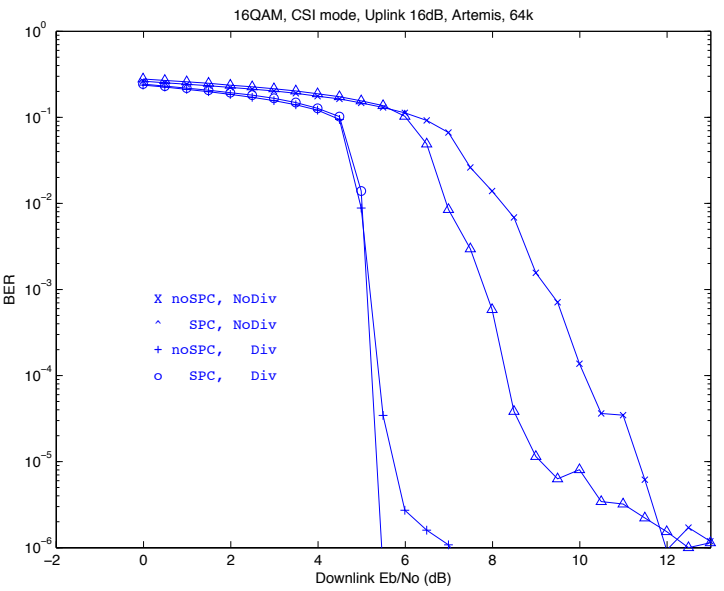

Fig. 6. 16QAM BER with high-scintillation Artemis fading.

useful to increase the erasure threshold value as the downlink SNR increased and the resulting erasure rate was between $5 \%$ and $25 \%$.

Given the method of detecting erasures based on the uplink fade level, the decoding algorithm was also modified to improve operation at higher downlink SNRs. This involved limiting the Es/No value given to the decoder, to about $10 \mathrm{~dB}$. The rationale for these extra decoder rules is straightforward: at large Es/No the decoder, taking the CSI at face value, believes the RF symbols are reliable, but in reality the CSI is imperfect since it depends on the FSO fade level and possibly the simple SPC check.

Figure 6 gives some comparisons for the highSI Artemis channel discussed in Section II. In this case larger codewords of $64 \mathrm{k}$ bits were used but this makes relatively little difference except for a somewhat sharper waterfall region. Rather than fitting a distribution, the actual Artemis fading channel samples were used in this case, again assuming ideal interleaving. The figure shows that the SPC code can provide a useful gain under high levels of fading when no uplink diversity is available. These results have used simplified SPC simulation. Rather than generating parity check bits, a genie aided approach has been used with the number of ones in the received symbol compared to the number of ones in the transmit symbol. In addition, for the
SPC modes, the uplink SNR has been adjusted by a factor of $\frac{M}{M+1}$ to account for the extra energy used in the parity symbol.

\section{CONCLUSIONS}

These simulations suggest that relatively simple interleaving and erasure approaches to mitigate uplink errors can provide very significant performance gains. Diversity is useful but not sufficient by itself. A large range of hybrid FSO-RF transmission and error detection/concealment options are possible and only a small number have been explored at this stage. Future work will include more realistic models of the uplink fading and noise, since these have a key role in determining overall performance, plus further investigation of diversity and coding options.

\section{Acknowledgements}

This project has been supported by the German Academic Exchange Service, DAAD. The authors would like to thank DLR colleagues Gianluigi Liva and Balazs Matuz for useful discussions.

\section{REFERENCES}

[1] ETSI, "Digital Video Broadcasting (DVB); Second generation framing structure, channel coding and modulation systems for Broadcasting, Interactive Services, News Gathering and other broadband satellite applications (DVB-S2)", Standard EN 302307 V1.3.1, March 2013

[2] R.Mata Calvo, P. Becker, D. Giggenbach, F. Moll, M. Schwarzer, M. Hinz, Z. Sodnik, "Transmitter Diversity Verification on Artemis Geostationary Satellite", Proceedings of the SPIE, Vol. 8971, Free-Space Laser Communication and Atmospheric Propagation XXVI, February 2014

[3] L. Andrews, R. Phillips, "Laser Beam Propagation through Random Media", SPIE Press, 2005

[4] N. Perlot, T. Dreischer, C. Weinert, J. Perdigues, "Optical GEO Feeder Link Design", Future Network and Mobile Summit 2012, 2012

[5] J. Romba, Z. Sodnik, M. Reyes, A. Alonso, and A. Bird,"ESA's bidirectional space-to-ground laser communication experiments", Proceedings of the SPIE, Vol. 5550, pp. $287-298,2004$

[6] M. Gregory, F. Heine, H. Kumpfner, R. Meyer, R. Fields, C. Lunde, "Tesat laser communication terminal performance results on 5.6 Gbit coherent inter satellite and satellite to ground links", International Conference on Space Optics ICSO 2010, 2010

[7] N.Perlot, J. Perdigues-Armengol, "Model-oriented availability analysis of optical GEO-ground links", Proceedings of the SPIE, Vol. 8246, 2012

[8] M. C. Valenti, Iterative Solutions Coded Modulation Library (ISCML), http://www.iterativesolutions.com/ 